\title{
Area surveillance by an underwater robot swarm based on virtual peromone field
}

\begin{abstract}
This paper proposes a virtual Pheromone inspired area surveillance method for underwater robot swarm. The whole system consists of a swarm of underwater robots and an underwater communication network. Robots in the swarm are unable to communicate directly, but can exchange data with the communication network by visiting communication nodes. We define two kinds of virtual Pheromone, i.e. the repel Pheromone secreted by robots when voyaging, and the attract Pheromone secreted by robots when it witnesses a target. Robots will voyage in the Area of Interest (AOI) driven by virtual Pheromone to search for targets. Simulation experiments have been carried out for an application scenario of using a swarm of underwater robots to search and track a target, showing that the performance of the virtual Pheromone based method is over that of the random search strategy.
\end{abstract}

Keywords: robots, virtual pheromone, humanitarian, underwater, optical light
Volume 2 Issue 3 - 2018

\author{
Guannan Li \\ Chinese Academy of Sciences, China
}

Correspondence: Guannan Li, Shenyang Institute of Automation, Chinese Academy of Sciences

Tel +8602 4239702 I 2, China, Email liguannan@sia.cn

Received: June 01, 2017 | Published: May II, 2018

\section{Introduction}

The main object of the research reported in this paper is to introduce a novel scheme to organize a swarm of underwater robot for area surveillance tasks. Area surveillance task is import in various applications, such as wreck salvageanti-mine operation, humanitarian mission and so on. These kinds of tasks are usually carried out with a single robot. However, it is beyond the ability of a single robot if the area of interest (AOI) is extensive. In this situation, we can use more than one robots working together to search for targets in the region, i.e. surveillance a region with a robot swarm. Area surveillance method has been widely researched for different kinds of robot swarms, especially for UAV swarm and UGV swarm. ${ }^{1-4}$ However, all these methods require reliable communication among the robots. Nowadays, the main hinder for cooperation among underwater robots is the lack of reliable communication. Available communication methods for underwater robots include acoustic communication and optical light communication. The former, acoustic communication is featured by long communication range but low in bandwidth, long communication delay and unreliable. Optical communication is fast so that can transfer large volumes of data within short time, but the sender and the receiver must be close enough. The performance of available communication methods for underwater robots cannot match that of the radio communication for UAVs and UGVs, making it hard to apply schemes for UAV and UGV swarms to underwater robot swarms directly.

On the other side, research on communication between an underwater robot and a set of fixed communication nodes has make some progress. Linked by underwater cables, these nodes compose a communication network. Underwater robots can visit these nodes to exchange data with the network. ${ }^{5,6}$ These researches make an inspiration for us, and lay the foundation for our work. In our swarm, robots will not communicate directly, but will visit and exchange data with the communication network. This kind of indirect communication is common for swarms in nature. ${ }^{7,8}$ The success of application of this kind of communication method encourages us to apply this method to our underwater robot swarm. Based on the communication network, we propose a virtual Pheromone based method to organize the underwater robot swarm. Each robot can secrete two kinds of Pheromone. When voyaging, a robot will secrete Repel Pheromone (RP). When see a target, a robot will secrete Attract Pheromone (AP). Robots are driven by the virtual Pheromone to avoid high density of RP while attract by AP. As robots cannot communicate real-timely, each robot maintains a unique Pheromone map. Robots will visit communication nodes periodically, uploading the Pheromone they secreted and downloading the updated Pheromone map.

\section{The contributions of this paper is}

a) Organizing an underwater robot swarm with a communication network, so that the constraints of direct communication among underwater robots are avoided.

b) Proposing a virtual Pheromone based method to drive robots to search for targets, whose performance is over the random walk method.

\section{Problem statement and assumptions}

The problem to solve is to surveillance an area with a swarm of underwater robots. Marine biologists want to know if a specific kind of marine animal exists in the AOI, so a swarm of underwater robots is deployed. The underwater robot swarm should report the existence of the animal, and also follow it to take videos. As discussed in section introduction, currently we do not have underwater communication device that is fast, reliable and long in communication range. However, communication between underwater robot and communication nodes has been tested successfully. So in our swarm, we abandon direct communication between a pair of underwater robots, but underwater robots can communicate with the communication nodes through optical communication method when a robot is visiting the communication nodes. As the swarm do not share real time communication, each robot need to make decision by itself, instead of waiting for command from the control center or a leader robot. This means we need to develop 
a distributed control strategy. We assume that a set of underwater communication nodes has been pre-deployed in the AOI, and these nodes are connected with underwater cable, making them a network that can share data real-time. The control center is connected with the underwater communication network, so that it can only get updated information when robots visiting communication nodes. The whole scenario is shown in Figure 1.

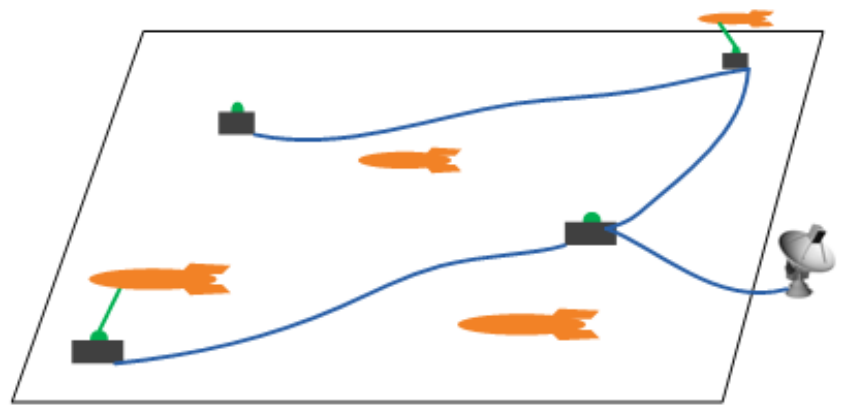

Figure I Swarm consists of robots and communication network.

In this scenario, we do not have enough robots to cover the whole AOI at the same time, so the swarm need to voyage in the AOI following certain strategy. We have no prior information about the targets, i.e. the number of targets, the position of targets and the behavior features of the targets. To simplify the design, the whole AOI is gridded. We assume that both the robots and the target are able to move from one grid to the neighbor grids at one simulation step. Each robot can detect the 9 grids around it, and for the targets, they will move continuously in the AOI.

\section{Virtual pheromone based behavior law}

We will define two kinds of virtual Pheromone in this section. Taken the virtual Pheromone into account, the gridded AOI becomes a Pheromone map for each robot. A set of behavior laws based on the Pheromone map is then defined. When a robot visits the communication node, both the robot and the communication network will update their Pheromone maps.

\section{Definition of virtual pheromone field}

In nature, with the help of Pheromone, social insects such as ants and termites can cooperate without direct communication, finally showing some kind of swarm intelligence. Individuals can "write" information into the environment by secreting Pheromone, and can also "read" information by sensing Pheromone. Virtual pheromone inspired methods have been widely researched in swarm robotics. Typically there are two ways to simulate virtual Pheromone. The first method is to establish communication among robots in the swarm, usually with radio communication devices, and design some type of data structures to represent the virtual Pheromone. ${ }^{9,10}$ This means robots still need to be able to communication directly, or even worse, they need to be able to communicate with all the other robots in the swarm. Considering current technology, we are unable to set up such a communication network in underwater robot swarms. The other method is to use some kind of chemistry or heat trace to mimic Pheromone. ${ }^{11-14}$ This method can be used in small arena, but not fit for robots voyaging in ocean.
In this research, we define a data structure called Pheromone map. A Pheromone map is a matrix, with each element corresponding to a grid of the AOI. The value of the element indicates the density of Pheromone. Each robot maintains a Pheromone map, and the communication network also maintains a Pheromone map. As robots in the swarm cannot communicate real time and directly, these Pheromone maps are not the same. The Pheromone maps will be uniformed when a robot visits a communication node. We define two kinds of virtual Pheromone, RP (Repel Pheromone) and AP (Attract Pheromone). When a robot is voyaging, it keeps secreting RP and RP will volatilize at certain speed. Namely when a robot enter a grid $(\mathrm{x}, \mathrm{y})$ , the density of RP in that grid is set as

$$
\mathrm{D}^{(\mathrm{x}, \mathrm{y})}=1
$$

The density of RP will be updated following:

$$
D_{\text {rp }}(t)=\left\{\begin{array}{cc}
1-v_{\text {vol }} t & D_{\text {rp }}(t-1)>0 \\
0 & D_{\text {rp }}(t-1) \leq 0
\end{array}\right.
$$

Here $\mathrm{v}_{\mathrm{vol}}$ is the volatize speed, which is in related with the number of grids and the number of robots, as:

$$
\mathrm{v}_{\mathrm{vol}}=\frac{\mathrm{m}}{\mathrm{N}}
$$

where $\mathrm{m}$ is the number of robots in the swarm and $\mathrm{N}$ is the number of grids.

This means in the ideal situation, when the swarm completes searching the whole area, the Pheromone density of the initial positions will become zero. When a robot detects a target, it will follow the target for a period. When it gives up following it, i.e. goes to visit a communication node, the robot will secrete AP at the point where it sees the target for the last time. So AP is similar to a marker for the position of the target. Let the position where the robot see the target be $\left(\mathrm{x}_{\mathrm{targ} \text { et }}, \mathrm{y}_{\mathrm{targ} \text { et }}\right)$, the process of a robot secreting AP is:

$$
\mathrm{D}_{\text {ap }}^{(\mathrm{x}, \mathrm{y})}=-1 \quad\left(\left\|(\mathrm{x}, \mathrm{y})-\left(\mathrm{x}_{\mathrm{t} \text { arg et }}, \mathrm{y}_{\mathrm{t} \text { arg et }}\right)\right\|<\mathrm{v}_{\text {voyage }} \mathrm{t}_{\text {visit }}\right)
$$

where $v_{\text {voyage }}$ the voyage is speed of the robot and $t_{\text {visit }}$ is the time spent by the robot to visit the nearest communication node. Then AP wills diffusion following the law below:

$$
D_{a p}(t)=\left\{\begin{array}{cc}
-1+v_{\text {diff }} & t \\
0 & D_{\text {ap }}(t-1)<0 \\
0 & (t-1) \geq 0
\end{array}\right.
$$

Where $\mathrm{v}_{\text {diff }}$ is the diffusion speed of AP.

It is obvious that the density of RP is within the range of $(0,1)$ and that of AP is $(-1,0)$. We can record two kinds of virtual Pheromone in the same Pheromone map. As the name implies, a robot will be attracted by AP while repelled by RP. From the perspective of Pheromone map, a robot tends to move to the grid with lower value.

\section{Behavior law}

The behavior law for each robot can be represented as a finite state machine, showing in Figure 2. The finite state machine contains four states, namely walk, follow, visit and report.

a. Detect the target.

b. Reach time threshold. 
c. Reach time threshold or realize another robot is also following.

d. Reach the nearest communication node.

e. Finish exchanging data.

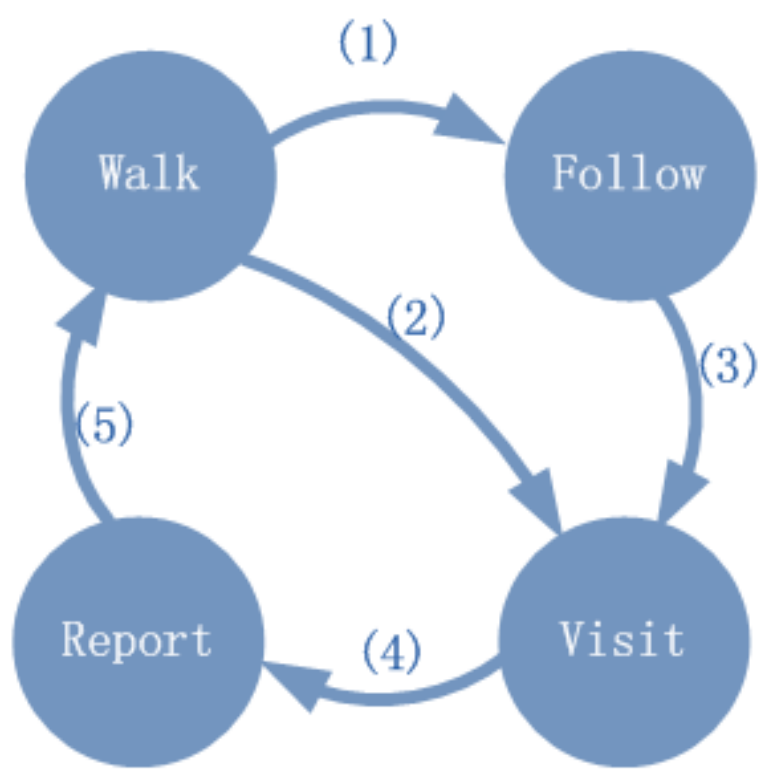

Figure 2 Behavior law for a robot in the swarm.

Walk: In state walk, the robot will check the grids around it and move to the grid with the lowest Pheromone density. In this state, the robot will update its Pheromone map according to Equation (2) and Equation (4). When the robot detects a target, it will transit to state Follow. If it has already walked for a while, reaching the time threshold, it will transit to state Visit.

Follow: Robot in state Follow can detect a target. It will move to the last grid of the target. If multiple robots are following the same target, each of them has $1 / 2$ chance to change state to Visit. When a robot changes state to Visit, it will secrete AP at the position when it see the target for the last time according to Equation (3), and will moving directly to the nearest communication node. If the robot has followed the target for a while, reaching the time threshold, it will also change to state visit.

Visit: In state visit, a robot will directly move to the nearest communication node. In this stage, it will stop secreting RP, but will keep updating AP. And it will also ignore any target during visiting.

Report: When a visiting robot reaches the communication node, it will change to state Report to exchange data with the communication network. After that, it will go back to state walk. By exchanging data, the Pheromone map of both the robot and the communication network will be updated. The data exchange process contains two stages. In the first stage, the robot will upload its Pheromone map, but only the part when it has secreted Pheromone. Taking the Pheromone map maintained by the communication network as the real environment, this means the robot secretes Pheromone into the environment. Then the robot will copy the whole Pheromone map from the network, so that it has the latest version of the whole Pheromone map.

\section{Simulation}

For simulation experiment, the AOI is scattered into $100 * 100$ grids. A swarm consists of 10 robots are deployed to search for a dynamic target. The endurance of the robots are 2000 simulation steps and initially all robots are fully charged. Robots have no prior information about the target. Once a target is detected, the robot should follow the target to collect data. After following for a period, it should give up following and go to visit a communication node to report the existence of the target so that the control center can make decision, such as sent out a boat. This period, also called a time threshold, is set as 50 simulation steps. So the metric of performance is the rate of following, i.e.:

$$
\mathrm{P}=\frac{\mathrm{T}_{\mathrm{f}}}{\mathrm{T}_{\text {total }}}
$$

Where $T_{f}$ is he total following time and $T_{\text {total }}$ is the total simulation time as 2000. This metric indicates how long the target has been followed by the swarm, also how much data has been collected. The performances of two methods are compared in the simulation experiment. One is the Pheromone based method proposed in this paper, and the other is the random search method. The random search method is a basic search strategy. In Walk state, a robot will choose its navigation direction randomly instead of according to the Pheromone density. To compare the performance of the two methods, we randomly generate 10 sets of trajectories for the target. For each trajectory, both the Pheromone based method and the random search method are repeated for 10 times. The experiment results are shown in Figure 3.

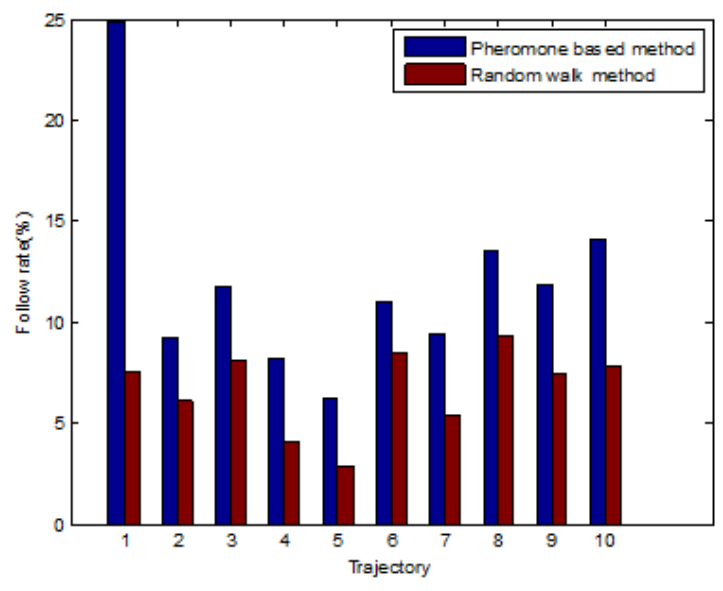

Figure 3 Simulation results.

From Figure 3, it is obvious that the performance of the Pheromone based method is over that of the random walk scheme. This is the result of using the two kinds of virtual Pheromone. With the application of $\mathrm{RP}$, robots have less chance to search the region that has already been searched, so the swarm tends to disperse in the region. Once the target is detected, the diffusion of AP indicates the region where targets have more chance to be, so that robots will be attracted to gather into the region, increasing the chance of the target been detected again. 


\section{Conclusion}

In this paper, we propose a scheme to organize a set of underwater robots into a swarm under the constraints that robots are unable to communicate directly. Robots can reach cooperation with indirect communication via a pre-deployed communication network. This design makes it possible to get a large scale underwater robot swarm scattered in an extensive area under current level of underwater communication technology. A virtual Pheromone based strategy is adopted to guide the swarm to surveillance the AOI. For a robot in the swarm, it can get information of the whole swarm by reading the Pheromone map and then make decision. As a result, the whole swarm emerges cooperation and the performance has been improved.

\section{Acknowledgments}

None.

\section{Conflict of interest}

Authors declare that there is no conflict of interest.

\section{References}

1. Saska M, Vonásek V, Chudoba J, et al. Swarm Distribution and Deployment for Cooperative Surveillance by Micro-Aerial Vehicles. Journal of Intelligent \& Robotic Systems. 2016;84(1-4):469-492.

2. An M, Wang Z, Zhang Y. Self-organizing strategy design and validation for integrated air-ground detection swarm. Jorunal of systems engineering and electronics. 2016;27(5):1018-1027.

3. Srivastava R, Budhraja A, Pradhan PM. An adaptive approach to swarm surveillance using particle swarm optimization. IEEE. 2016: pp.37803783.

4. Saldana D, Alitappeh RJ, Pimenta LCA, et al. Dynamic perimeter surveillance with a team of robots. International Conference on Robotics and Automation. 2016: p. 5289-5294.
5. Sun K, Wang X, Li Z. Application of underwater wireless optical communication technology in seafloor observatory network. Boletin Tecnico. 2017;55(13):456-464.

6. Li M, Guo S, Guo J, et al. Development of a biomimetic underwater microrobot for a father-son robot system. Microsystem Technologies. 2017;23(4):849-861.

7. Bonabeau E, Dorigo M, Theraulaz G. Swarm intelligence: from natural to artificial systems. Oxford University Press; 1999.

8. Goss S, Beckers R, Deneubourg J L, et al. How Trail Laying and Trail Following Can Solve Foraging Problems for Ant Colonies. Behavioural Mechanisms of Food Selection. 1990. p. 661-678.

9. Moon WS, Jang JW, Kim HS, et al. Virtual pheromone map building and a utilization method for a multi-purpose swarm robot system. International Journal of Control Automation \& Systems. 2015;13(6):1446-1453.

10. Pinciroli C, Lee-Brown A, Beltrame G. A Tuple Space for Data Sharing in Robot Swarms. Eai International Conference on Bio-Inspired Information and Communications Technologies. 2016. p. 287-294.

11. Russell RA. Heat trails as short-lived navigational markers for mobile robots. IEEE International Conference on Robotics and Automation. 1997;4:534-3539.

12. Sugawara K, Kazama T, Watanabe T. Foraging behavior of interacting robots with virtual pheromone. International Conference on Intelligent Robots and Systems. 2004;3:3074-3079.

13. Garnier S, Tache F, Combe M, et al. Alice in Pheromone Land: An Experimental Setup for the Study of Ant-like Robots. Swarm Intelligence Symposium. 2007. p. 37-44.

14. Fujisawa R, Dobata S, Sugawara K, et al. Designing pheromone communication in swarm robotics: Group foraging behavior mediated by chemical substance. Swarm Intelligence. 2014;8(3):227-246. 